\title{
Software Livre Twine: ensino de programação Web por meio da criação de jogos educacionais
}

\author{
Walter R.B. Carvalho ${ }^{1}$, Carla L. Rodriguez ${ }^{2}$, Denise H. Goya ${ }^{2}$, \\ Mirtha L.F. Venero ${ }^{2}$, Rafaela V. Rocha ${ }^{2}$ \\ ${ }^{1}$ Universidade Federal de São Carlos (UFSCar) - São Carlos, SP, Brasil \\ ${ }^{2}$ Universidade Federal do ABC (UFABC) - Santo André, SP, Brasil \\ waltercarvalho@estudante.ufscar.br, \{c.rodriguez, denise.goya, \\ mirtha.lina, rafaela.rocha\} dufabc.edu.br
}

\begin{abstract}
Several studies report the successful use of games engines and software like Scratch for teaching programming. The free software Twine, used for creating games and interactive narratives, does not require advanced knowledge, but its use in the classroom has not been explored and reported. In this article, we describe a methodology for using Twine to teach Web programming, creating an Educational Game (EG). The methodology includes: (1) Study and bibliographical research, (2) Course Planning, (3) Implementation and data collection, and (4) Evaluation of results. The participants created six EGs, which were evaluated using analysis criteria, and self-assessment and pre and post-tests to measure the knowledge acquired.
\end{abstract}

Resumo. Vários estudos relatam o uso com sucesso de games engines e softwares como Scratch no ensino de programação. O software livre Twine, usado na criação de games e narrativas interativas, não exige conhecimento avançado, porém seu uso em sala de aula não tem sido explorado e relatado. Neste artigo é descrita uma metodologia de uso do Twine para ensino de programação Web, criando um Jogo Educacional (JE). A metodologia contém: (1) Estudo e pesquisa bibliográfica, (2) Planejamento do curso, (3) Implementação e coleta de dados e (4) Avaliação dos resultados. Os participantes criaram seis JEs, que foram avaliados usando critérios de análise, além de uma autoavaliação e de pré e pós-testes para mensurar o conhecimento adquirido.

\section{Introdução}

A educação no século XXI, diante de mudanças sociais e desenvolvimento tecnológico, passou por diversas transformações para ser capaz de educar as novas gerações com base nas necessidades de cada década. Nesse contexto, o surgimento de novas mídias e tecnologias possibilita novas práticas culturais e educacionais e contribui para o processo de inclusão digital. Dentre as diferentes tecnologias usadas em práticas pedagógicas estão os jogos educacionais, que são capazes de criar engajamento, compartilhar conhecimentos e desenvolver novas habilidades (Squire, 2007), além da possibilidade de integração com outras mídias. Além desses jogos, são relatados com sucesso no ensino de programação os usos de ambientes visuais de programação (como Scratch e Alice) (Rodriguez et al., 2015; Souza e Castro, 2016; Knittel et al., 2017; Weintrop et al., 2018) e de game engines (Kafai e Burke, 2015; Santos et al., 2018). 
VIII Congresso Brasileiro de Informática na Educação (CBIE 2019)

Anais dos Workshops do VIII Congresso Brasileiro de Informática na Educação (WCBIE 2019)

Jenkins (2009) aponta o potencial de narrativas transmídias por meio da exploração dos melhores aspectos de cada mídia, possibilitando que os processos de ensinoaprendizagem não se mantenham unicamente na educação formal e promovendo a inclusão digital e a inserção das pessoas em comunidades de educação informal. Em comunicação com diferentes mídias digitais, a ferramenta Twine ${ }^{1}$, que é livre e open-source, vem sendo utilizada, principalmente, por causa de: i) aplicação pedagógica em educação informal, por meio da construção de narrativas; e ii) potencial de ensino de programação em linguagens Web (Deitos, Franco e Peres, 2018).

Nesse contexto, este trabalho apresenta a metodologia proposta, as atividades desenvolvidas e os resultados de um curso de extensão realizado com professores e interessados na criação de jogos educacionais. O objetivo da pesquisa é avaliar uma abordagem de ensino de linguagens de desenvolvimento $W e b$ por meio da criação de jogos educacionais explorando a ferramenta Twine. O objetivo do curso de extensão foi o ensino do uso da ferramenta Twine e de linguagens de desenvolvimento Web (HTML, CSS e JavaScript), além de abordar conceitos de games e narrativas interativas criando um jogo educacional.

Este trabalho está organizado como a seguir. A seção 2 apresenta os conceitos de narrativas interativas, um breve histórico sobre o Twine e trabalhos relacionados, seguido da metodologia, na seção 3. Na seção 4 são descritos os resultados e discussões, incluindo a avaliação de aprendizagem. Na seção 5 são apresentadas as conclusões.

\section{Fundamentos Teóricos}

Nesta seção são descritos os principais conceitos sobre as narrativas interativas e jogos eletrônicos, a ferramenta Twine e os trabalhos relacionados.

\subsection{Narrativas interativas e games}

Narrativa interativa é uma experiência digital na qual o usuário, por meio de tomadas de decisões, consegue impactar o rumo de uma história de forma não-linear, seja no papel de um personagem como protagonista ou manipulando uma realidade como um elemento externo (Riedl e Bulitko, 2013). Isso possibilita novos pontos de vistas e experiências praticamente únicas a cada usuário. Observa-se que a liberdade é limitada para evitar uma descaracterização da própria história, apesar de diferentes caminhos, esses estão atrelados à todas as possibilidades que não levem a uma história fora do proposto inicialmente (Audi e Regis, 2011). Outros fatores que impedem possibilidades infinitas são: (1) linguagem, já que as ações estão limitadas à criatividade do roteirista, e (2) limitações técnicas na construção do jogo (Audi e Regis, 2011).

As narrativas interativas são eficazes dentro de um contexto transmidiático, sendo possível trazer a textualidade de livros para jogos, facilitado por meio das características imersivas e interativas dessa mídia (Riveiros, 2018). Além disso, as histórias podem ser criadas em diferentes gêneros, tais como, novelas visuais, adventures gráficos e filmes interativos. Um dos principais benefícios da transposição desses elementos é seu uso em contextos educacionais, gerando hábitos de leitura e transmitindo conceitos de diferentes áreas do conhecimento (Taborda e Alves, 2015).

\footnotetext{
${ }^{1}$ https://twinery.org, último acesso julho de 2019.
} 
VIII Congresso Brasileiro de Informática na Educação (CBIE 2019)

Anais dos Workshops do VIII Congresso Brasileiro de Informática na Educação (WCBIE 2019)

\subsection{Twine}

A ferramenta Twine, desenvolvida em 2009 e popularizada em 2012, foi planejada para construção visual de narrativas interativas com hyperlinks e texto em suas passagens, sendo extremamente acessível e intuitiva para pessoas sem conhecimentos em programação (Friedhoff, 2013). O Twine é baseado em HTML, possui diversos formatos de história, e também possibilita o uso de CSS e JavaScript para personalizar e implementar as interações, layout e acesso a diferentes recursos multimídia.

Os formatos de histórias foram incluídos no Twine 2.0 e oferecem diferentes experiências para o desenvolvedor. Estes formatos alteram o layout de interação do usuário, e oferecem diferentes mecânicas pré-estabelecidas. Entre os formatos de histórias mais populares estão: (1) Harlowe: oferece uma aparência e interações formatadas para ser possível a criação de uma história sem conhecimentos em linguagens Web; (2) Snowman: oferece um layout mínimo, que exige sua customização em HTML e CSS; e (3) SugarCube: oferece recursos de layout, como menus para salvar e reiniciar o jogo, mas exige conhecimentos avançados de CSS e JavaScript.

A "Revolução Twine", movimento criado por desenvolvedores de jogos LGBTQ, colaborou com a difusão da ferramenta por utilizá-la em grande escala na construção de narrativas autobibliográficas, temáticas queer e experimentações com formas diversas de contar histórias (Bragança, Mota e Fantini, 2016).

\subsection{Trabalhos Relacionados}

Na literatura são apresentados mapeamentos sistemáticos e revisões de pesquisas com o uso de ferramentas específicas que exploram a criação de games (tais como, Scratch, Alice, Takkou) e uso de motores de jogos no ensino de programação (por exemplo, PyGame, GameMaker, Construct2) (Medeiros, Silva e Aranha, 2013; Silva, 2017). O objetivo dessas pesquisas está no ensino de introdução à lógica de programação, pensamento computacional, estrutura de algoritmos, programação orientada a objetos e aprendizado de linguagens de programação (Medeiros, Silva e Aranha, 2013; Silva, 2017). Nelas, o foco maior é a introdução à lógica de programação e pensamento computacional já que muitas ferramentas e motores de jogos tem suporte a linguagens próprias de script, com exceções como Pygame (Python) e Construct2 (JavaScript).

Em iniciativas educacionais, observa o uso do Twine especialmente em cursos de escrita. Chen e outros (2019) usaram o Twine na familiarização de ficções interativas e hyperlinks e um bot para Twitter no desenvolvimento da escrita de texto digitais, em um curso que integra algoritmos e humanidades. Engström, Brusk e Erlandsson (2018) usaram o Twine com a ferramenta de prototipação de jogos Deig ${ }^{2} \mathrm{em}$ um curso de escrita de diálogos voltado para jogos. Hahn (2016) apresentou o Twine em um curso de escrita colaborativa, no qual os grupos de alunos organizaram a produção de uma narrativa interativa, via e-mail ou Google Docs, e posteriormente realizaram a hierarquização dos trechos pelo Twine. Clark e Baxter (2018) usaram o Twine em aulas da graduação de Biologia para reforço dos conteúdos, nas quais a construção foi realizada em grupos de jogos temáticos de microbiologia. De forma geral, a literatura carece de estudos sobre o uso de Twine com foco no ensino de programação, visto que outras ferramentas têm sido bem relatadas.

${ }^{2}$ https://deig.se/, último acesso julho de 2019. 
VIII Congresso Brasileiro de Informática na Educação (CBIE 2019)

Anais dos Workshops do VIII Congresso Brasileiro de Informática na Educação (WCBIE 2019)

\section{Metodologia}

A metodologia proposta e usada neste trabalho seguiu os passos: 1) Estudo e pesquisa bibliográfica; 2) Planejamento e preparação do curso; 3 ) Implementação do curso e coleta de dados; 4) Avaliação dos resultados. Essa é uma simplificação do modelo ADDIE (Analysis, Design, Develop, Implement, Evaluate) (Morrison, 2010) para criação de materiais instrucionais, sendo que as etapas de design e desenvolvimento foram substituídas por planejamento e preparação. Os passos são detalhados a seguir.

\subsection{Estudo e pesquisa bibliográfica}

Foram levantados os principais conceitos sobre narrativas, jogos e Twine (descritos na seção 2.1 e 2.2 e abordados no curso), bem como, trabalhos relacionados (2.3).

\subsection{Planejamento e preparação do curso}

Foi planejado o curso de extensão sobre criação de jogos com o Twine, com total de 12 horasaulas, tendo como público-alvo professores e interessados na criação de jogos educacionais. O minicurso teve a participação de integrantes do Laboratório de Informações em Rede e Tecnologias Educacionais (LIRTE ${ }^{3}$ ), da Universidade Federal do ABC, e dos projetos Diversão Séria e ++C\&TPM, para organizar, oferecer e atuar como monitores do minicurso. Um aluno elaborou e liderou a oferta do curso. Como materiais foram criados e usados slides e modelos de layout do Twine, desenvolvidos pelo aluno e com a supervisão de quatro docentes.

As aulas contam com conteúdo teórico do Twine e das linguagens de programação $W e b$, seguido de parte prática e um horário reservado para dúvidas sobre o projeto. O projeto proposto é uma tarefa para grupos de 2 a 4 pessoas, iniciado no primeiro dia de aula, que deve ser desenvolvido durante a semana pelos integrantes dos grupos, com espaço para desenvolvimento e resolução de dúvidas em sala de aula, e apresentados no último dia. $\mathrm{O}$ Quadro 1 mostra o objetivo de aprendizagem e a divisão das unidades instrucionais com seus respectivos temas e conteúdos.

\section{Quadro 1. Objetivos de aprendizagem e sequências instrucionais.}

\begin{tabular}{|c|c|}
\hline \multicolumn{2}{|c|}{ Curso: "Criação de games e narrativas interativas com o Twine" } \\
\hline $\begin{array}{l}\text { Objetivos de } \\
\text { aprendizagem }\end{array}$ & $\begin{array}{l}\text { Ensino de conceitos de linguagem de programação Web (HTML, CSS e JavaScript) para } \\
\text { aplicação em jogos e narrativas interativas na ferramenta Twine. }\end{array}$ \\
\hline \multicolumn{2}{|c|}{ Primeira Unidade Instrucional } \\
\hline Tema & Introdução a narrativas interativas, Twine e HTML \\
\hline Conteúdo & $\begin{array}{l}\text { História de narrativas interativas, narrativas interativas e transmídias, história do Twine, } \\
\text { familiarização com a interface da ferramenta (criação de passagens), estrutura e } \\
\text { propriedades de HTML (cabeçalhos de seção, parágrafos, imagens, estilos e formatação). }\end{array}$ \\
\hline \multicolumn{2}{|c|}{ Segunda Unidade Instrucional } \\
\hline Tema & Introdução à CSS e JavaScript \\
\hline Conteúdo & $\begin{array}{l}\text { Sintaxe, formas de implementação e propriedades da linguagem de folha de estilo CSS } \\
\text { (fontes, plano de fundo, margem, preenchimento, altura e largura, estilização de }\end{array}$ \\
\hline
\end{tabular}

${ }^{3}$ http://pesquisa.ufabc.edu.br/lirte/, último acesso julho de 2019. 
VIII Congresso Brasileiro de Informática na Educação (CBIE 2019)

Anais dos Workshops do VIII Congresso Brasileiro de Informática na Educação (WCBIE 2019)

\begin{tabular}{|l|l|}
\hline \multicolumn{1}{|l|}{} & $\begin{array}{l}\text { cabeçalhos, rodapés e corpo de texto, formatos de história do Twine, estruturas e } \\
\text { propriedades de JavaScript (variáveis numéricas e não-numéricas, vetores, números } \\
\text { aleatórios, funções e condicionais). }\end{array}$ \\
\hline Terceira Unidade Instrucional \\
\hline Tema & Outras propriedades de JavaScript e JQuery \\
\hline Conteúdo & $\begin{array}{l}\text { Definição e implementação de frameworks, funcionalidades do JQuery que alteram HTML } \\
\text { e CSS, eventos de teclado com JQuery. }\end{array}$ \\
\hline
\end{tabular}

Considerando a duração do curso e a adaptação destas linguagens para o Twine, o curso ofereceu enfoque nas propriedades mais relevantes de cada linguagem e que eram significativas para a construção do jogo. Também tiveram casos de uso de propriedades adaptadas pelo Twine para uma implementação mais simples, como no caso de links internos, criação de variáveis e condicionais. Nesses casos, foram ensinados tanto sua implementação com o formato de história Harlowe 3.0.2 quanto nas linguagens de desenvolvimento Web.

\subsection{Implementação do curso e coleta de dados}

O curso foi idealizado para ser realizado em três dias (total de 12 horas-aulas). As aulas são ministradas por meio de datashow em um laboratório com computadores individuais, com Linux e acesso online ao Twine, por meio do navegador Web Firefox. A avaliação dos objetivos de aprendizagem ocorreu por meio de dois questionários, um aplicado na primeira aula e outro na última. $O$ código do projeto de jogo educacional também foi analisado, com avaliação do uso das propriedades de linguagens $W e b$, o que pode indicar uma possível familiarização das propriedades utilizadas. Além disso, no último dia foi realizada uma autoavaliação sobre a percepção de aproveitamento do curso e aprendizado das linguagens. A mesma teve a escala Likert como formato de resposta, em uma escala que varia de discordo completamente até concordo completamente, na faixa de 1 a 5 , no qual o valor pode ser posteriormente comparado aos resultados do questionário e do uso de propriedades no jogo desenvolvido em grupo. Nesta autoavaliação também foram usadas perguntas abertas, possibilitando uma maior compreensão sobre a percepção dos alunos com o curso. Os questionários (pré, pós-testes e estão disponíveis: http://pesquisa.ufabc.edu.br/lirte/diversaoseria/twine2019.

\subsection{Avaliação dos resultados}

Em relação aos testes (pré e pós), as perguntas são referentes: quatro a HTML, três a CSS e três a JavaScript. Para a análise dos códigos e uso dos recursos, foram criados três critérios de uso de propriedades para cada linguagem (Quadro 2).

\section{Resultados e Discussões}

O curso foi ofertado em três sábados no mês de junho de 2019 para, inicialmente, 21 alunos (com 15 concluintes). Além do professor, dois monitores apoiaram os alunos durante as aulas.

\subsection{Perfil dos participantes}

Os sujeitos da pesquisa inscritos foram selecionados de um total de 192 alunos, com base em análise curricular, em um recorte de pessoas que não conhecem ou conhecem pouco as linguagens de desenvolvimento $W e b$ e com interesse na área da educação. 
VIII Congresso Brasileiro de Informática na Educação (CBIE 2019)

Anais dos Workshops do VIII Congresso Brasileiro de Informática na Educação (WCBIE 2019)

Os dados das inscrições possibilitaram a identificação dos participantes do curso, com idades variando entre 21 e 52 anos (conforme Figura 1a). A turma foi composta por 11 educadores e 4 estudantes com interesses na área de educação, destacando-se a presença de professores de ensino médio no curso (Figura 1b), e em sua maioria, os professores de outros cursos atuam como professor de apoio pedagógico e implementação de tecnologias em práticas pedagógicas.

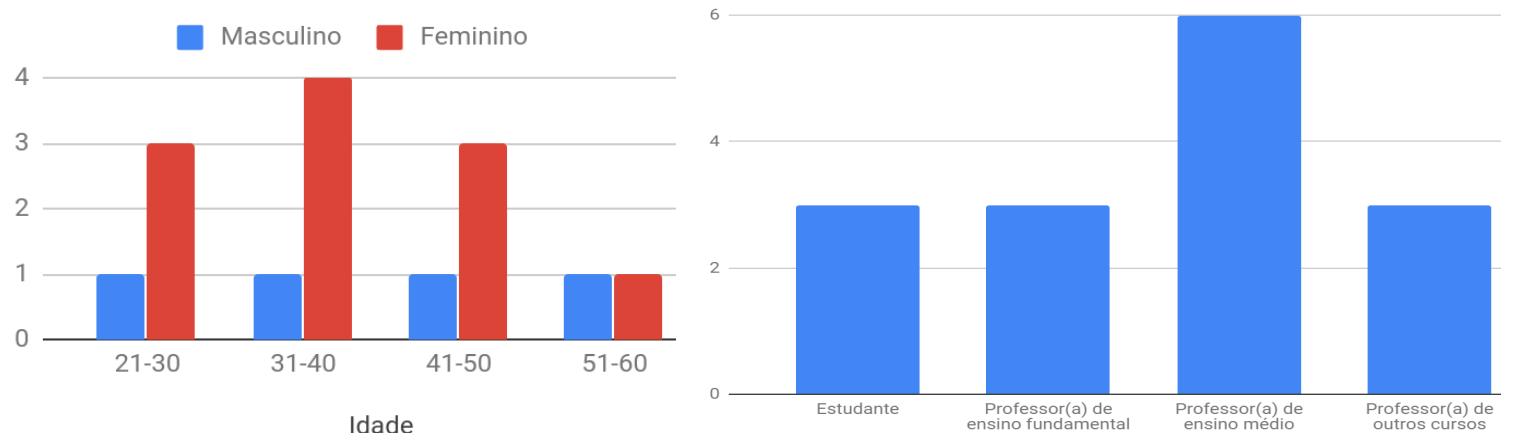

Figura 1. (a) Idade dos participantes do curso e (b) Atuação dos professores por nível de ensino.

Em relação à formação e ocupação, seis são professores de ensino médio (licenciados em Matemática (2), Química (2), Letras e Pedagogia), dois de ensino fundamental (pedagogos), três de outros cursos (formados em Ciências da Comunicação, Letras e Pedagogia), e três são estudantes de graduação (Logística e Ciência e Tecnologia (2)).

\subsection{Coleta de dados}

O pré-teste foi respondido por 21 alunos e o pós-teste respondido por 15 alunos, em aproximadamente 20 minutos cada. Seis protótipos de jogos educacionais foram desenvolvidos de forma independente pelos grupos durante o curso. Os grupos, formados de duas a quatro pessoas, ficaram livres para escolher a temática, e os conceitos e estruturas de programação. Sendo essas estruturas selecionadas mais de acordo com o jogo idealizado, ao invés de explorar os recursos disponíveis das linguagens de programação Web. O professor e monitores ajudaram de forma pontual, principalmente em casos de erros no código. Foram construídos jogos voltados ao ensino básico e médio, sendo: dois com temática multidisciplinar (tais como, literatura, história e biologia), um de matemática, um de física, e dois voltados ao desenvolvimento emocional, conforme podem ser vistos na Figura 2.
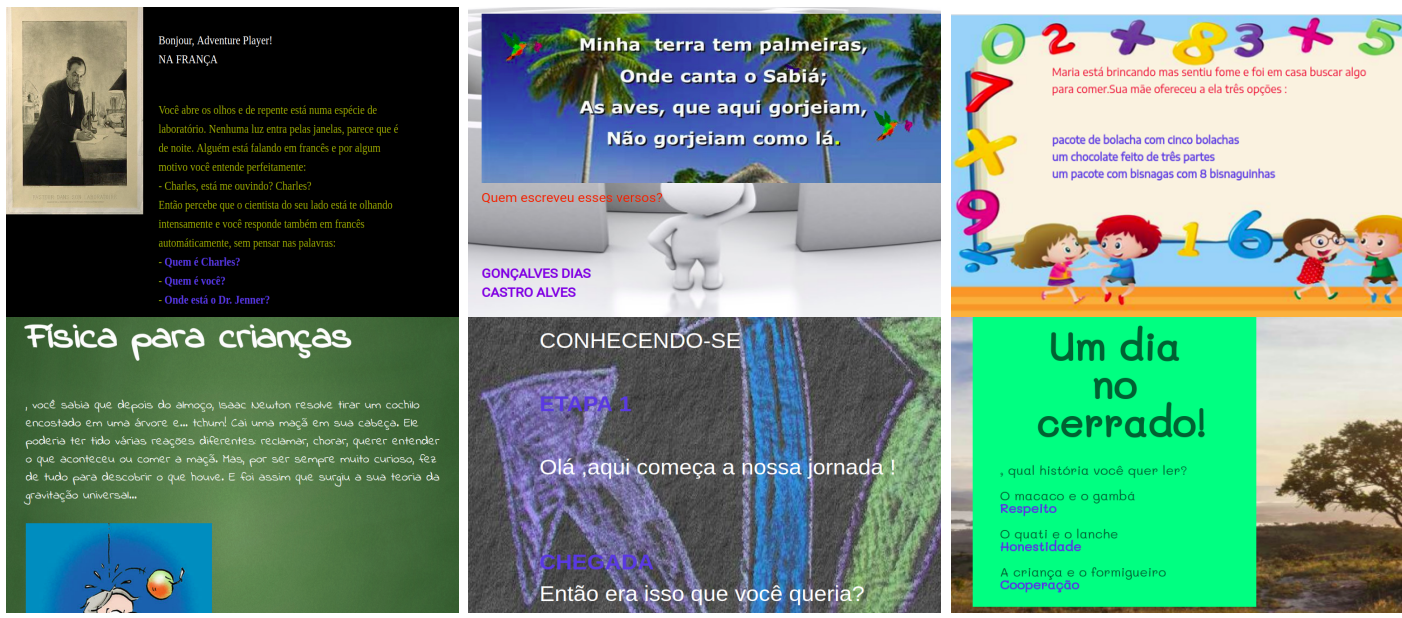

Figura 2. Captura de tela dos projetos desenvolvidos durante o curso. 
VIII Congresso Brasileiro de Informática na Educação (CBIE 2019)

Anais dos Workshops do VIII Congresso Brasileiro de Informática na Educação (WCBIE 2019)

\subsection{Análise dos dados da avaliação dos objetivos de aprendizagem}

As Figuras 3 e 4 apresentam os gráficos dos resultados do pré e pós-testes, referente à avaliação dos objetivos de aprendizagem. Na Figura 3, observa-se uma diminuição na escolha da alternativa "não sei", de forma que as escolhas de alternativa correta e errada tiveram crescimento similares, sendo que a alternativa correta teve um crescimento um pouco maior (Figura 3b).
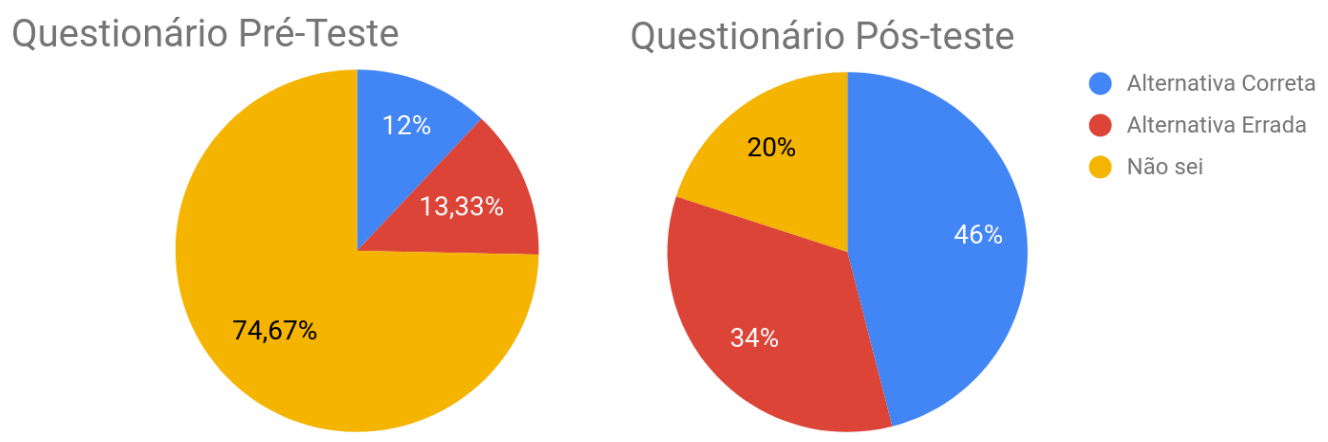

Figura 3. Resultados dos questionários (a) pré-teste e (b) pós-teste.

Em relação aos acertos por linguagem $W e b$, Figura 4, nota-se um crescimento maior de acerto das perguntas referentes à HTML, uma possível explicação é o fato das propriedades não terem uma sintaxe tão complexa para iniciantes no assunto, da mesma forma para a linguagem CSS. Em relação às possíveis explicações para a dificuldade dos alunos com JavaScript (Figura 4b) estão: a facilidade de implementação de variáveis e condicionais usando o código do Twine, impedindo assim o uso e a familiarização de códigos JavaScript, maior complexidade na síntese do JavaScript para iniciantes e a limitação do tempo de curso.
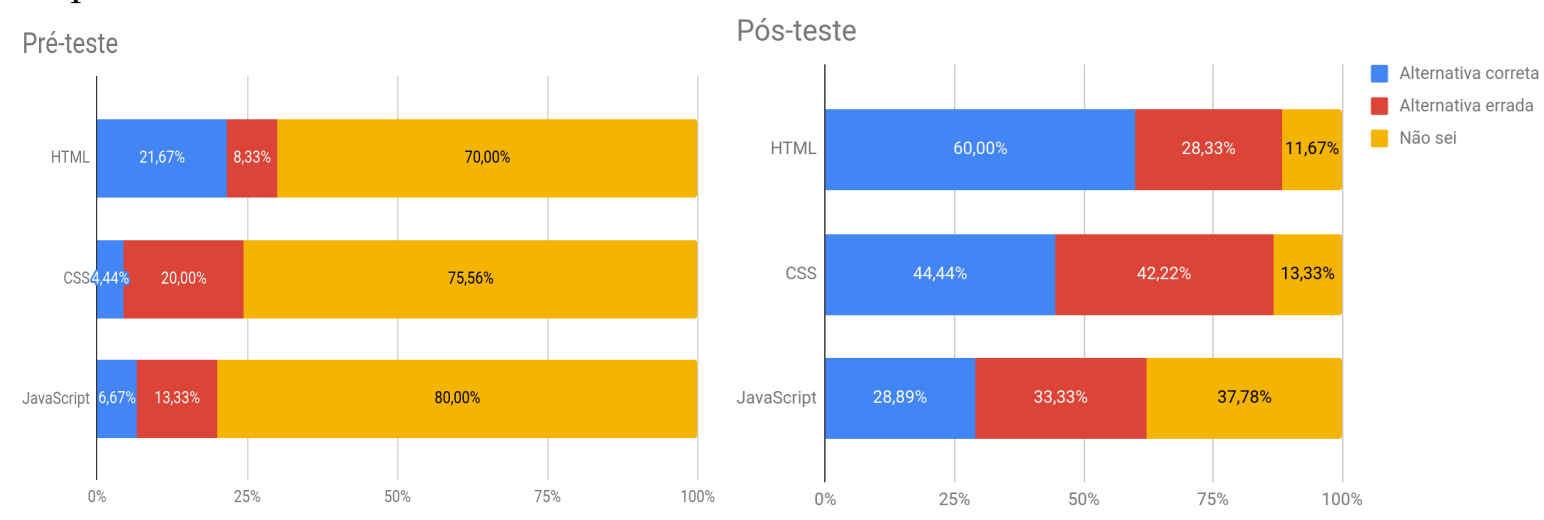

Figura 4. Comparativo dos resultados dos questionários com base na linguagem Web (a) pré-teste e (b) pós-teste.

O uso dos critérios de análise dos projetos possibilitou uma maior compreensão dos principais conceitos utilizados por cada grupo, podendo indicar os elementos considerados mais significativos na construção de narrativas interativas com o Twine (Quadro 2). O alto número de critérios atendidos de CSS, comparado a outros critérios, indica que a parte estética foi considerada o ponto mais importante na construção de um jogo, possivelmente pela percepção de que a originalidade de cada jogo ocorre com sua aparência, sendo que o menor uso de espaçamento e margem pode ser explicado pela dificuldade de implementação na construção do layout. Os baixos índices de uso de HTML, em comparação ao de CSS, 
VIII Congresso Brasileiro de Informática na Educação (CBIE 2019)

Anais dos Workshops do VIII Congresso Brasileiro de Informática na Educação (WCBIE 2019)

indicam que os grupos preferiram alterar a aparência do jogo por meio da folha de estilo da história, com poucas exceções, e não foi dada muita atenção ao uso de cabeçalho e rodapé.

O uso dos critérios de JavaScript ocorreu especialmente por meio do uso de variáveis, e em menor escala condicionais e declaração de botões. Isso pode ser explicado pela facilidade na implementação de variáveis pelo Harlowe, e em muitos casos, na solicitação do nome do jogador, utilizando a variável "nome" com um viés de intimidade; bem como do sistema de passagem por hyperlink, não precisando de botões. No caso da tag "<script $>$ ", seu uso é mais complexo e com diferentes possibilidades, desde programar botões e o teclado para um avatar andar até a integração com um banco de dados (por exemplo, planilha do google sheets), porém, só foi usada a programação de botões.

Quadro 2. Critérios de análise dos projetos.

\begin{tabular}{|c|c|c|}
\hline Linguagem & Critério de análise & Porcentagem de uso \\
\hline \multirow{3}{*}{ HTML } & Formatação do texto com HTML & $17 \%$ \\
\cline { 2 - 3 } & Manipulação dos elementos semânticos & $33 \%$ \\
\cline { 2 - 3 } & Uso de atributos em tags HTML & $33 \%$ \\
\hline \multirow{3}{*}{ CSS } & Propriedades relacionadas à estilização do plano de fundo & $100 \%$ \\
\cline { 2 - 3 } & Propriedades relacionadas à estilização do texto e hyperlinks & $100 \%$ \\
\cline { 2 - 3 } & Propriedades relacionadas aos espaçamentos e margens & $33 \%$ \\
\hline \multirow{3}{*}{$\begin{array}{c}\text { JavaScript e } \\
\text { código Twine }\end{array}$} & Uso de variáveis (declarada em Twine) & $67 \%$ \\
\cline { 2 - 3 } & Uso de condicionais (declarada em Twine) & $17 \%$ \\
\cline { 2 - 3 } & Uso de botões (declarados com JavaScript) & $17 \%$ \\
\hline
\end{tabular}

Os resultados da percepção dos alunos diante de seu aprendizado corroboram com os dados dos questionários de pré e pós-teste e com os dados do uso de linguagens nos jogos. Os alunos indicaram uma maior facilidade no aprendizado de HTML e CSS, por usarem mais essas linguagens durante o curso (Figura 4). Sendo que uma explicação para a falta de confiança no aprendizado, de uma parte da turma, foi o tempo de curso (relatado em uma questão aberta), já que em 12 horas-aulas não pôde ser reforçado o uso de códigos Web essenciais, ocasionando uma menor segurança na aprendizagem.

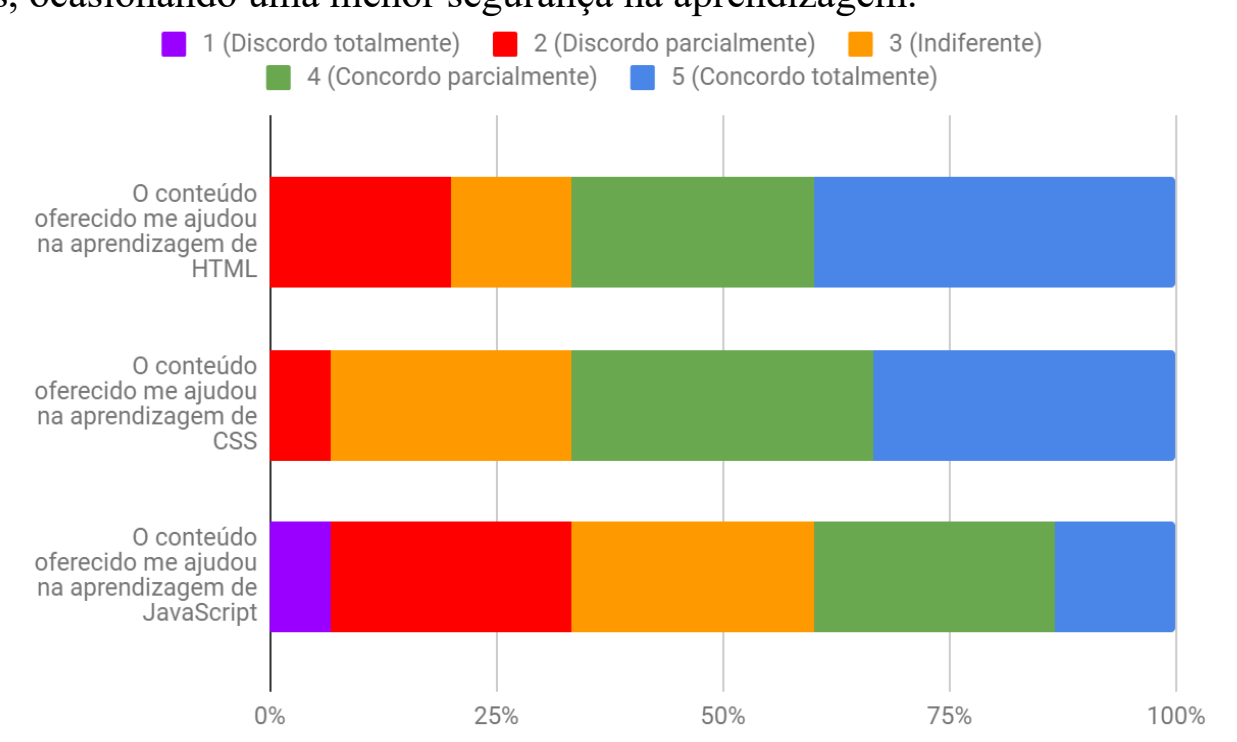

Figura 4. Percepção dos alunos sobre aprendizagem de cada linguagem Web. 
VIII Congresso Brasileiro de Informática na Educação (CBIE 2019)

Anais dos Workshops do VIII Congresso Brasileiro de Informática na Educação (WCBIE 2019)

Em relação às perguntas abertas, foram registradas opiniões positivas referentes à proposta de ensinar linguagens Web por meio do material oferecido no curso e da construção de um projeto em grupo usando o Twine; em relação aos aspectos do curso que poderiam ser melhorados, os comentários se voltaram ao número de aulas, que não foram suficientes para o aprofundamento no conteúdo das três linguagens, e a possibilidade de mais tempo voltado à parte prática, em especial CSS e JavaScript.

\section{Conclusões}

Apesar da popularização da ferramenta Twine em 2012, poucas iniciativas investigaram e relataram o seu potencial pedagógico multidisciplinar, principalmente, comparado a outras ferramentas como Logo e Scratch que são bem conhecidas na área de Informática e Educação. O presente trabalho propôs uma metodologia e investigou o uso do Twine no processo de ensino-aprendizagem de conceitos de linguagens de desenvolvimento $\mathrm{Web}$, por meio da criação de jogos educacionais. Considerando a diversidade de formas que narrativas interativas podem tomar, o uso de diferentes propriedades em um ambiente visual possibilita a criação de jogos originais e o reforço do conteúdo aprendido.

Os resultados mostraram que a proposta de produzir um jogo em Twine, aliado a um roteiro de conteúdo teórico e prático de linguagens de desenvolvimento $\mathrm{Web}$, é uma estratégia para despertar o interesse e oferecer elementos introdutórios destas linguagens. Entretanto, há a simplificação de algumas propriedades, como no caso de variáveis e loops, introduzidas ao usuário em um formato de pseudocódigo, com foco mais no conceito do que à sintaxe das linguagens tradicionais.

Além disso, há a necessidade de mais aulas para o desenvolvimento destes conteúdos, já que apesar da viabilidade de alguns formatos do Twine possibilitarem a construção de um jogo apenas com a escrita da narrativa, perde-se no processo de aprendizagem das linguagens $W e b$, visto que os participantes querem produzir um jogo completo, estando mais focados nessa criação do que na experimentação dos conceitos e estruturas de programação.

Considerando os aspectos multidisciplinares a serem explorados, é recomendável em trabalhos futuros, a avaliação da ferramenta no desenvolvimento da capacidade de escrita narrativa e dissertativa, bem como estudar com mais detalhes o desenvolvimento do pensamento computacional. É recomendado também que o curso tenha mais horas-aula para que os alunos possam explorar os conceitos e estruturas de programação, bem como, criar um jogo completo.

\section{Referências}

Audi, G. M., Regis, F. (2011) Jogos Narrativos: aproximação da narração da cultura oral com os jogos de videogame. Comunicação \& Inovação, 12(22), p. 72-78.

Bragança, L., Mota, R., Fantini, E. (2016) Twine Game Narrative and discussion about LGBTQ representation. In SBGames, p. 937-946.

Chen, Y., Chen, Z., Gumidyala, S., Koures, A., Lee, S., Msekela, J., Remash, H., Schoenle, N., Albright, S. D., Rebelsky, S. A. (2019) A Middle-School Code Camp Emphasizing Digital Humanities. Proceedings of the 50th SIGCSE, p. 351-357.

Clark, J., Baxter, R. (2018) From Passive Players to Active Developers: Undergraduate Biology Students Developing Their Own Digital Learning Game with Twine. Journal of Applied Instructional Design, 7(1), p. 7-16. 
VIII Congresso Brasileiro de Informática na Educação (CBIE 2019)

Anais dos Workshops do VIII Congresso Brasileiro de Informática na Educação (WCBIE 2019)

Deitos, F, Franco, M, Peres, A. (2018) Ditado Digital: um jogo para auxílio da escrita no Ensino Fundamental. In XXIX SBIE, p. 834-843.

Engström, H., Brusk, J., Erlandsson, P. (2018) Prototyping Tools for Game Writers. The Computer Games Journal, 7(3), 153-172.

Friedhoff, J. (2013) Untangling Twine: A Platform Study. DiGRA conference. p. 1-10.

Hahn, R. (2016) Collaborative creative writing in the L2 classroom using the software Twine. In Proceedings of the 6th Future of Education International Conference, p. 137-142.

Jenkins, H. (2009) Cultura da Convergência. 2a. Ed. SP: Aleph.

Kafai, Y. B., Burke, Q. (2015) Constructionist gaming: Understanding the benefits of making games for learning. Educational psychologist, 50(4), p. 313-334.

Knittel, T.F.; Garcia, M.S.S.; Iglesias, K.; Trujillo, D.S. (2017) Scratch: uma linguagem de construção interativa de competências matemáticas. In SBGames, p. 1016-1019.

Medeiros, T. J., Silva, T. R., Aranha, E. H. S. (2013) Ensino de programação utilizando jogos digitais: uma revisão sistemática da literatura. RENOTE, 11(3), p. 1-10.

Morrison, G.R. (2010) Designing Effective Instruction, 6th ed. John Wiley \& Sons.

Riedl, M. O.; Bulitko, V. (2013) Interactive narrative: An intelligent systems approach. Ai Magazine, 34(1), p. 67-77.

Riveiros, S. H. B. (2018) Alice no País dos Videogames: um estudo da narrativa em jogos digitais. In Proceedings of SBGames, p. 19-27.

Rodriguez, C., Zem-Lopes, A. M., Marques, L., Isotani, S. (2015) Pensamento Computacional: transformando ideias em jogos digitais usando o Scratch. In XXI WIE, p. $62-71$.

Santos, C.P.; Silva, D.R., Ferreira, G., Silveira, M.G.F. (2018) Desenvolvimento de Jogos Digitais como uma Estratégia para Despertar Novos Talentos: um relato de experiência. In XXIV WIE, p. 129-136.

Silva, J. C. D. (2017) Ensino de Programação para alunos do Ensino Básico: Um levantamento das pesquisas realizadas no Brasil. TCC (Licenciatura em Ciência da Computação), UFPB.

Souza, S., Castro, T. (2016) Investigação em programação com Scratch para crianças: uma revisão sistemática da literatura. In Workshops do V CBIE, p. 1078-1086.

Squire, K. D. (2007) Games, Learning and Society: Building a Field. Educational Technology, p. 51-55.

Taborda, A. G., Alves, P. K. (2015) Visual Novel: a evolução do gênero e sua aplicação para desenvolver o hábito da leitura. In Proceedings of SBGames 2015, p. 483-492.

Weintrop, D., Hansen, A.K., Harlow, D.B., Franklin, D. (2018) Starting from Scratch: Outcomes of Early Computer Science Learning Experiences and Implications for What Comes Next. In ACM Conference on International Computing Education Research, p. 142-150. 